\title{
Sample-to-Answer Diagnostic System for the Detection of Circulating Histones in Whole Blood
}

\author{
Micaela L. Everitt ${ }^{1}$, David J. Boegner ${ }^{1}$, Konstantin G. Birukov², and lan M. White ${ }^{1, *}$
}

1. Fischell Department of Bioengineering, University of Maryland 20740, United States

2. Anesthesiology Department, University of Maryland Medical Center 21201, United States

*Email: ianwhite@umd.edu

\section{Electronic Supporting Information}

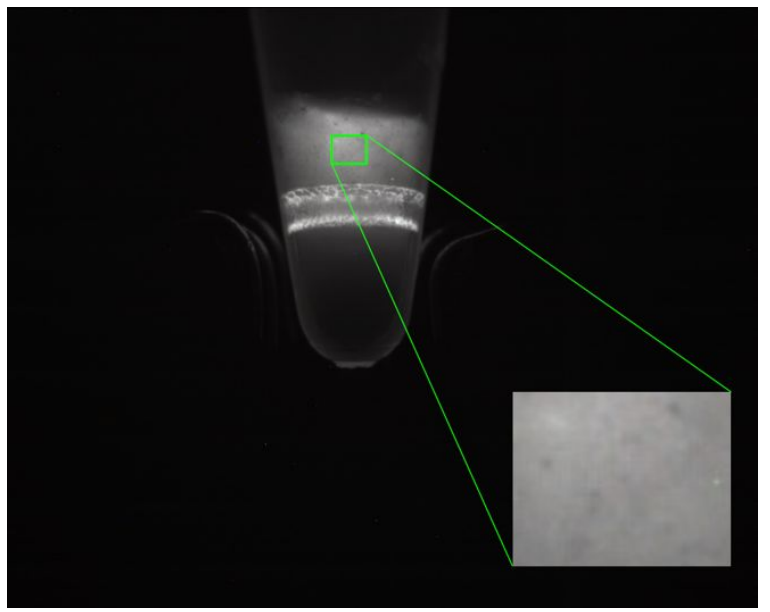

Figure S1. Image of reaction tube taken by portable imaging device. An algorithm selects a box in the fluorescent region and quantifies the grayscale intensity.

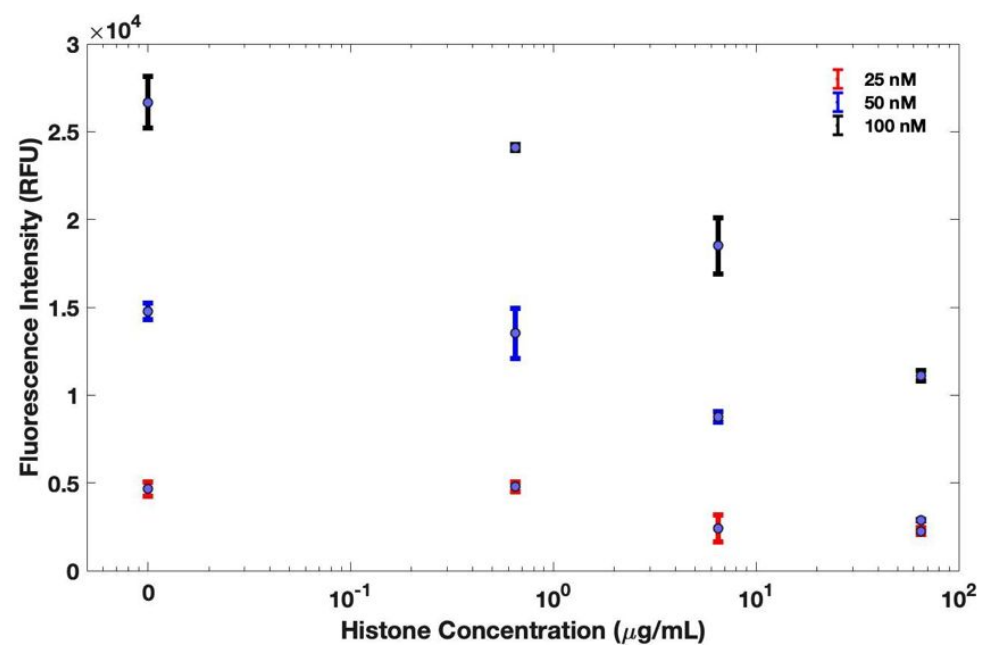

Figure S2. Measured fluorescence intensity versus histone concentration for varying concentrations of double-stranded DNA in the reaction. 

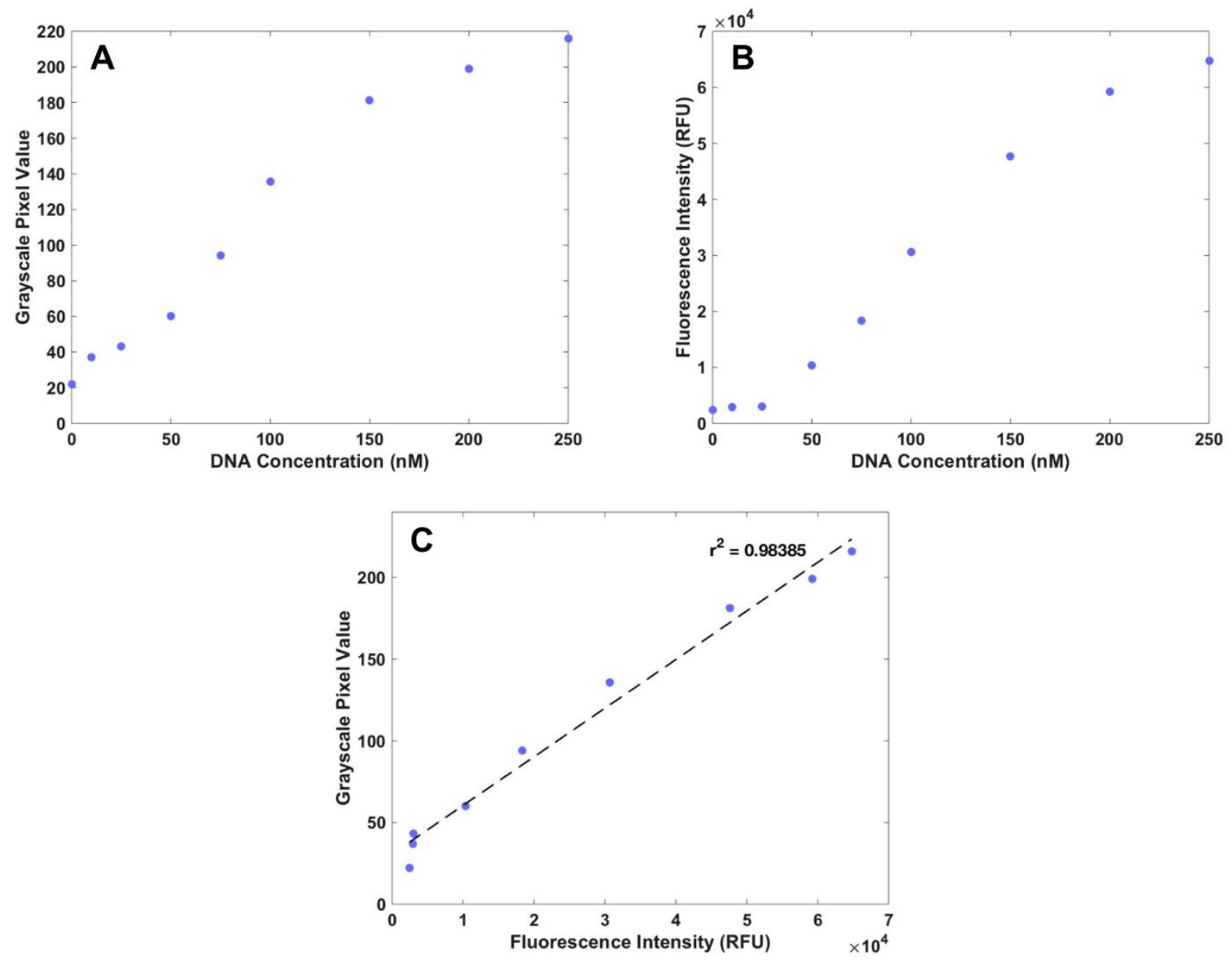

Figure S3. Comparison of benchtop plate reader (Synergy LX from BioTek) to portable fluorescence reader. Varying $147 \mathrm{bp}$ DNA is added with 1x EvaGreen and resulting fluorescence is measured in both devices. The portable fluorescence reader has slightly improved detection limits over the gold standard comparison. (A) Fluorescent measurements produced from portable fluorescence reader. (B) Fluorescent measurements produced from benchtop plate reader. (C) Comparison of resulting measurements from the portable fluorescence reader and benchtop plate reader. 


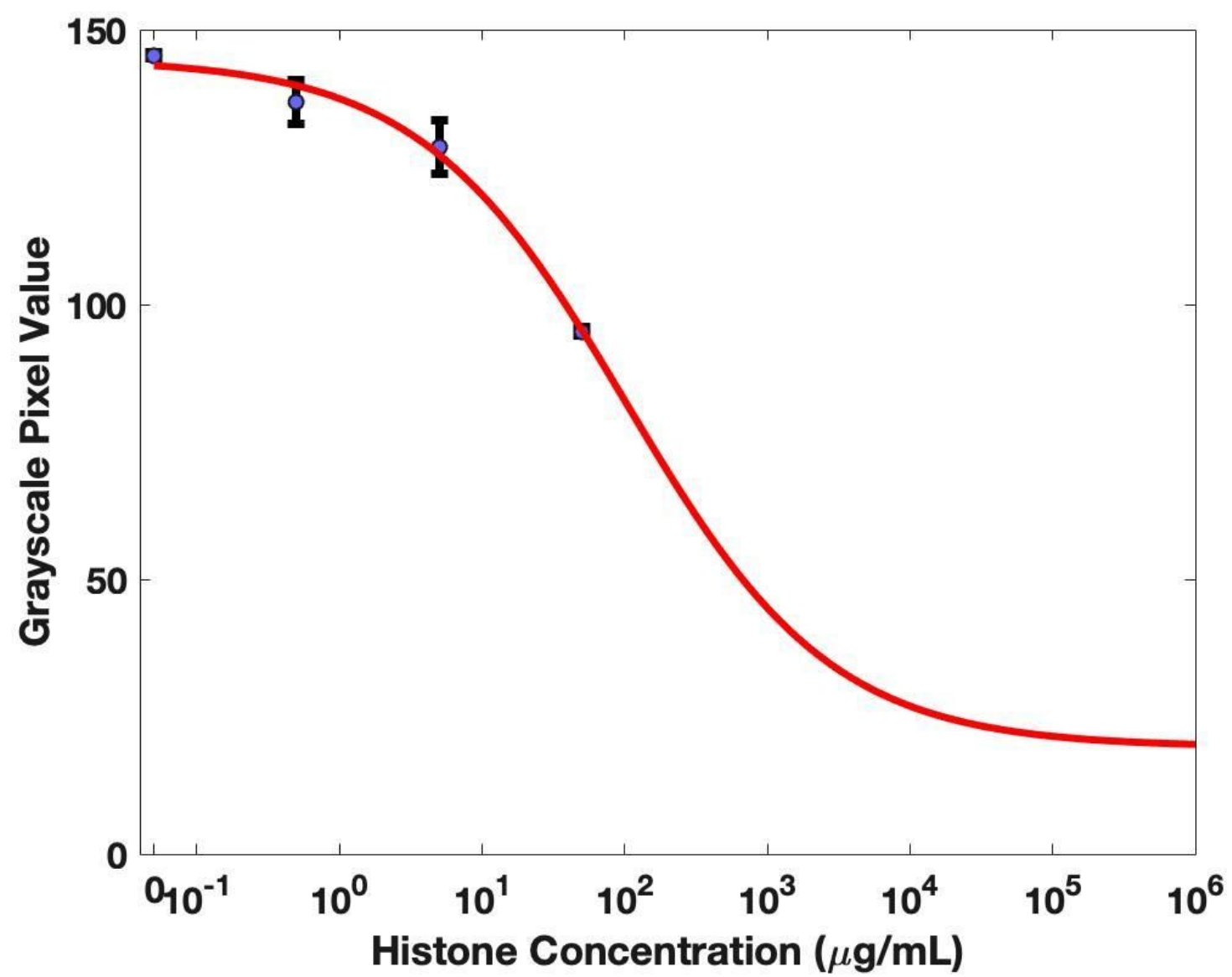

Figure S4. Reverse sigmoidal curve fit. Using a reverse sigmoidal curve fit in MATLAB and assuming a grayscale pixel value of a non-fluorescent sample as histone concentration approaches infinity, we modeled the curve for histone levels above what we tested experimentally. The extrapolated upper limit of detection is about $0.1 \mathrm{~g} / \mathrm{mL}$ histone octamers in blood, several magnitudes above clinically relevant values.

\section{Limit of detection calculations.}

Full definitions and calculations of limit of detection. $A$ is an abbreviation for analytical sensitivity which is the slope between the blank and lowest positive concentration. The standard deviations are calculated from the triplicates at the blank and the lowest positive datapoint.

$$
\begin{gathered}
\text { IUPAC LoD }=\left(3 \times \sigma_{\text {blank }}\right) / \mathrm{A} \\
\text { ISO LoD }=1.645\left(\sigma_{\text {blank }} / \mathrm{A}\right)+1.645\left(\sigma_{\text {lowest positive }} / \mathrm{A}\right) \\
\sigma_{\text {blank }}=0.6326 \quad \sigma_{\text {lowest positive }}=4.0183 \\
\mathrm{~A}=(145.3434-136.8975) /(0.5-0)=16.8917
\end{gathered}
$$

